\title{
Interferometric microstructured polymer optical fiber ultrasound sensor for optoacoustic endoscopic imaging in biomedical applications
}

\author{
Daniel Gallego*a , David Saéz-Rodriguéz ${ }^{\mathrm{b}}$, David Webb ${ }^{\mathrm{b}}$, Ole Bang ${ }^{\mathrm{c}}$, Horacio Lamela ${ }^{\mathrm{a}}$ \\ ${ }^{a}$ Optoelectronics and Laser Technology Group, Universidad Carlos III de Madrid, Av. de la \\ Universidad 30, Leganes,Spain; ${ }^{\mathrm{b}}$ Aston Institute of Photonic Technologies, Aston University, \\ Birmingham, UK;'DTU Fotonik, Dept. of Photoniks Engineering, Technical University of Denmark, \\ DK-2800 Kgs. Lyngby, Denmark
}

\begin{abstract}
We report a characterization of the acoustic sensitivity of microstructured polymer optical fiber interferometric sensors at ultrasonic frequencies from $100 \mathrm{kHz}$ to $10 \mathrm{MHz}$. The use of wide-band ultrasonic fiber optic sensors in biomedical ultrasonic and optoacoustic applications is an open alternative to conventional piezoelectric transducers. These kind of sensors, made of biocompatible polymers, are good candidates for the sensing element in an optoacoustic endoscope because of its high sensitivity, its shape and its non-brittle and non-electric nature. The acoustic sensitivity of the intrinsic fiber optic interferometric sensors depends strongly of the material which is composed of. In this work we compare experimentally the intrinsic ultrasonic sensitivities of a PMMA mPOF with other three optical fibers: a singlemode silica optical fiber, a single-mode polymer optical fiber and a multimode graded-index perfluorinated polymer optical fiber.
\end{abstract}

Keywords: Microstructured polymer optical fiber, interferometry, ultrasound detectors, optoacoustic detectors

\section{INTRODUCTION}

Recent advances in ultrasound and optoacoustic imaging techniques for clinical applications demands for miniaturized and wide bandwidth ultrasonic probes[1]. It has been presented in 2012 the images of internal organs in vivo using a 2.5$\mathrm{mm}$ diameter optoacoustic and ultrasonic dual-mode endoscope[2]. In order to extend the use of these kind of dual mode endoscopes to complement the intravascular ultrasound (IVUS), adding to the morphological information the specificity of the optoacoustic imaging, it is necessary to miniaturize the probe to be fitted in less than $1 \mathrm{~mm}$ to pass through thin vasculature. Moreover, for a practical IVUS/IVPA catheter is mandatory to have a wideband ultrasonic detector up to $50 \mathrm{MHz}$ with enough sensitivity despite the necessary miniaturization. The optical detection of ultrasound using optical fiber sensors can provide the compactness, sensitivity and bandwidth required for this application.

Our group, previously, has demonstrated that ultrasonic sensitivity of an interferometric single mode polymer optical fiber sensor (SMPOF) is one order of magnitude higher than a silica counterpart[3]. However, these SMPOF are not easily commercially available and its performance in terms of loss and coupling light into is very poor what makes them impractical for real implementation. The lack of a good single mode POF has forced to use multimode POF to take advantage of the sensitivity improvement at the cost of a degradation in the visibility of the interference resulting in noise when both arms of the interferometer are not matched or in a Fabry-Perot interferometer.

In contrast, microstructured polymer optical fiber (mPOF), exhibiting the same acoustic sensitivity, presents moderate low loss at visible wavelength regime and can be made endlessly single-mode. In this contribution, we present an ultrasonic characterizations of an intrinsic mPOF sensor based on PMMA.

In this contribution we present an experimental comparison of acoustic sensitivity between interferometric mPOF sensor and other three interferometric sensors based on other fiber optics: single-mode silica optical fiber (SMSOF), single mode POF and multi-mode graded-index perfluorinated polymer optical fibers (GIPOF). All sensors are designed for detection of optoacoustic wave sources with dimensions between $15 \mathrm{~mm}$ and less than $0.1 \mathrm{~mm}$ what corresponds to ultrasonic frequencies in the range from $100 \mathrm{kHz}$ to $10 \mathrm{MHz}$.

*dcgallego@ing.uc3m.es; phone+34 624 8758; portal.uc3m.es/portal/page/portal/grupos_investigacion/optoelectronics 


\section{EXPERIMENTAL SETUP}

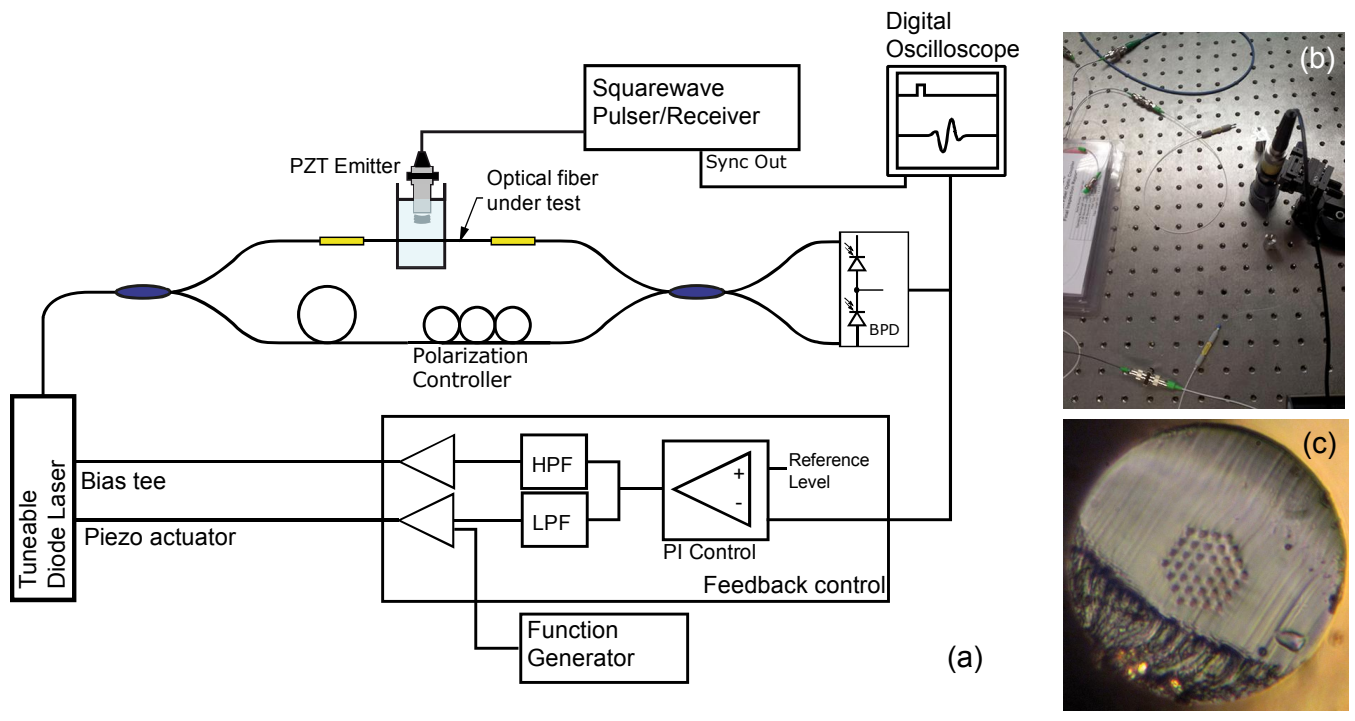

Figure 1. (a) Schematic diagram of the experimental setup for sensitivity characterization of the optical fiber sensor. The light from a Littman external cavity tunable diode laser centered at a wavelength of $850 \mathrm{~nm}$ is coupled into an optical fiber Mach-Zehnder interferometer. The polarization controller is used to guarantee equal state of polarization at the output of both arms maximizing the visibility of the interference. The calibrated PZT emitter and the optical fiber are inside a tube filled with water to ensure a consistent acoustic coupling. A feedback control acts over the emission wavelength of the laser to maintain the interferometer in the quadrature point. (b) Photograph of the optical fiber test arrangement. (c) Micrograph of the end facet of the PMMA mPOF with outside diameter $125 \mu \mathrm{m}$.

An ultrasonic wave incident on an optical fiber produces a phase modulation due to the induced strains and the strainoptic effect. The phase shift is converted to an optical intensity variation using a Mach-Zehnder interferometer. The measurement system used for the characterization of the acoustic sensitivity of one straight segment of an optical fiber is presented in figure 1a. An external cavity tunable diode laser (Sacher, Lion TEC-500), with central wavelength at $850 \mathrm{~nm}$, is used as laser source for a Mach-Zehnder interferometer. In the reference arm a polarization controller is used to equal the polarization state in both arms. The output of the interferometer is measured with a balanced photodectector (BPD) with $100 \mathrm{MHz}$ of bandwidth. The output voltage is divided between a digital oscilloscope which captures the signal and a laser wavelength feedback control that fixes the working point of the interferometer. The reference arm is longer than the measurement arm in the interferometer so a change in the output wavelength of the laser effectively produces a phase shift between both arms of the interferometer. The feedback control consists in a proportional-integral (PI) filter followed by two filters that divide the signal spectrum. The output of the low-pass filter goes to a gain control and then feed a piezo actuator that moves the mirror of the external cavity. This piezo actuator has a resonant frequency of $1.5 \mathrm{kHz}$. The low pass filter cut-off frequency is settle to $300 \mathrm{~Hz}$. The output of the high pass filter also goes to another gain control and through a bias-tee modulates the diode laser current. The lower frequencies perturbations, for instance, changes in temperature and pressure, are compensated with the piezo actuator while the high frequency perturbations, i.e, audible acoustic waves that can excite the resonance of the piezo actuator, are outweighed by changes in the laser current. This system avoids excessive diode laser current changes that would have an noticeable impact in the interference visibility. A function generator signal can be added to the piezo actuator input allowing the test of the interference visibility at any instant. The system is able to work automatically controlled by a computer.

Unfocussed immersion transducers with central resonant frequencies of $1 \mathrm{MHz}$ and $10 \mathrm{MHz}$ (Panametrics V303 and V327) were used as ultrasonic emitters to test the optical fibers. These transducers were excited using a square pulse generator (Panametrics 5077PR). The amplitude of the square pulse was varied between $100 \mathrm{~V}$ and $400 \mathrm{~V}$ at steps of $100 \mathrm{~V}$. These PZT transducers have been previously calibrated with a $100 \mathrm{MHz}$ wideband ultrasonic detector. Water is used to ensure consistent and reproducible acoustic coupling between the emitter and the optical fiber segment under test.

We have characterized the acoustic sensitivity of a mPOF and other three different optical fibers, one made of silica and the others built out of different polymers. The MPOF is a three ring made of polymethyl methacrylate (PMMA) with a 
hole diameter to pitch ratio of about 0.5 (fig 1.c) thus is single-mode. The silica optical fiber (SM800-5.6-125) is single mode at $830 \mathrm{~nm}$ and has a cladding diameter of $125 \mu \mathrm{m}$. It is jacketed with an acrylate coating what increases the outside diameter (OD) to $245 \mu \mathrm{m}$. The third optical fiber is a PMMA single-mode POF (from Paradigm Optics, Vancouver, WA, USA). It has a cladding diameter of $125 \mu \mathrm{m}$ and a core diameter of $8 \mu \mathrm{m}$. This fiber has been designed to have a cut-off wavelength of less than $750 \mathrm{~nm}$. Finally, the last optical fiber characterized is a perfluorinated graded-index polymer optical fibers (GIPOF-50, from Chromis Fiberoptics, Warren, NJ, USA). It has a cladding diameter of $490 \mu \mathrm{m}$ and a core diameter of $50 \mu \mathrm{m}$. This multimode fiber offers lower attenuation compared to the SMPOF.

\section{RESULTS AND DISCUSSION}
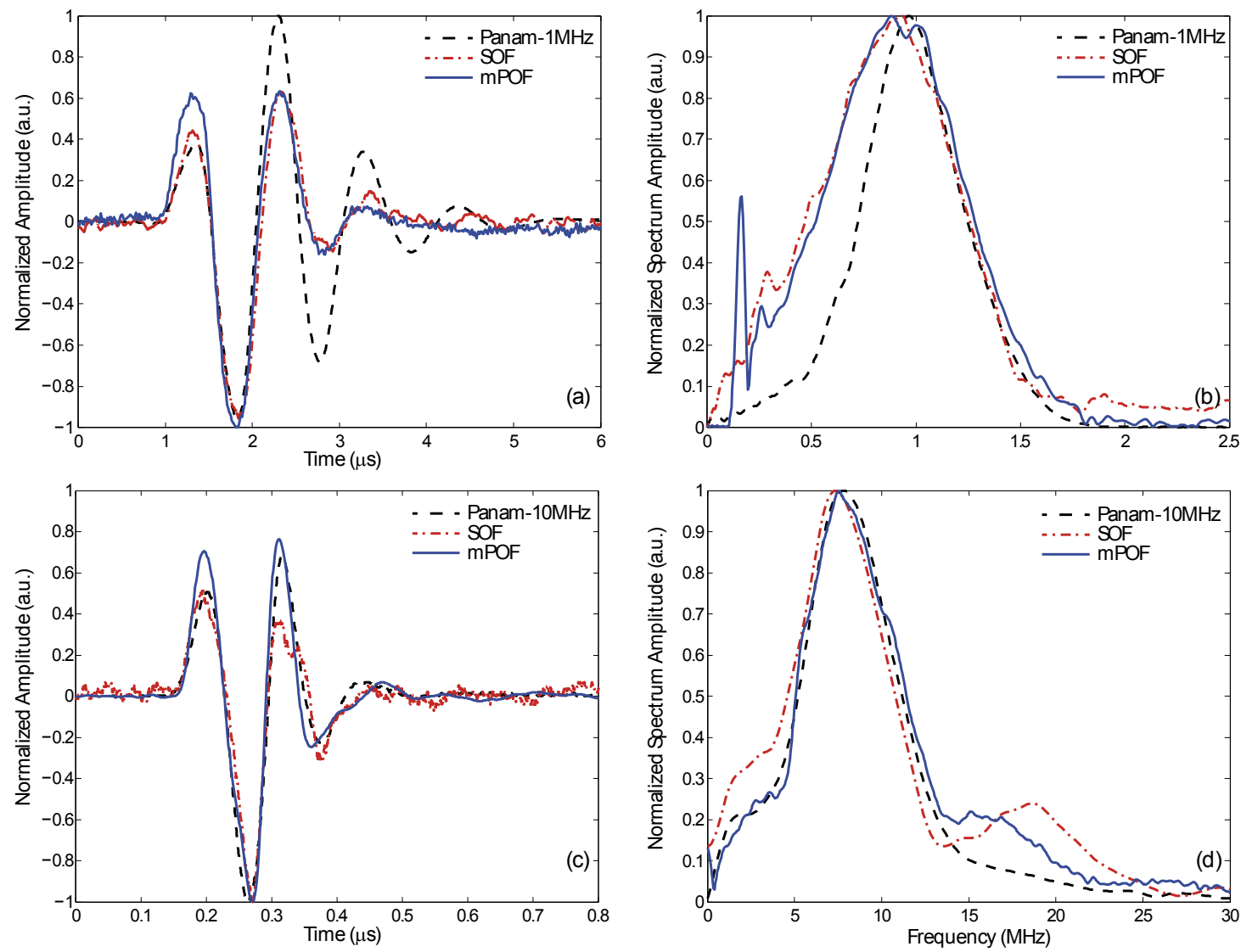

Figure 2. (a) Comparison between the normalized amplitude of the $1 \mathrm{MHz}$ pulsed signals measured by a resonant $1 \mathrm{MHz}$ unfocussed Panametrics transducer, the silica optical fiber interferometer and the mPOF interferometer. (b) Normalized spectrum amplitude of the $1 \mathrm{MHz}$ signals. (c) and (d) Normalized amplitude and spectrum of the $10 \mathrm{MHz}$ pulsed signals measured by a $100 \mathrm{MHz}$ wideband transducer (WAT-13), the silica optical fiber and the mPOF.

We have measured ultrasonic signals from PZT emitters using all the fibers under test. Figure 2 presents the normalized amplitude of $1 \mathrm{MHz}$ and $10 \mathrm{MHz}$ temporal signals and its spectrums for a perpendicular incidence of the ultrasonic waves. Signals for the other two polymer optical fibers can be seen in [4]. The temporal signals of both fibers are similar in shape, but the SOF one is much more noisy due to its lower sensitivity compared to mPOF. Contrary to the single mode fiber MORPOF03, the MPOF has low attenuation at $850 \mathrm{~nm}$ what permits to have an interference with the same visibility as using SOF. This is an important advantage, since the high attenuation of the SMPOF has prevented their use in the final application of detecting optoacoustic generated waves. The GIPOF-50 has good performance at few megahertz but shows noticeable reverberations at higher frequencies. Moreover, because it is a multimode fiber the interference are going to be inherently more noisy than the mPOF when there is a mismatch in the length of the arms of the interferometer. This has an negative impact in the minimum phase detectable by the interferometer. The spectrum of the optical fiber signals covers all frequencies contained in the emitted pulses. The PZT emitter spectrum (dashed black) at 
$1 \mathrm{MHz}$ corresponds to the signal captured by other identical resonant transducer and it is narrow band contrary to the optical fiber sensors. However, the spectrum at $10 \mathrm{MHz}$ was captured by the $100 \mathrm{MHz}$ wideband transducer.
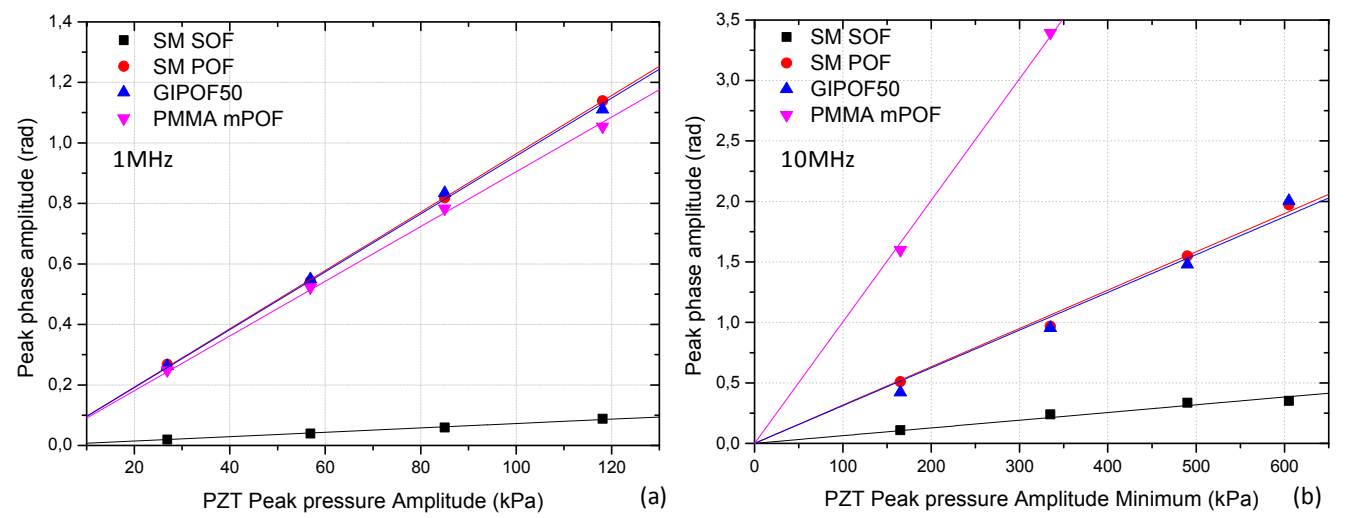

Figure 3. Relation between pulse amplitude pressure of the 1 and $10 \mathrm{MHz}$ emitters and the phase shift induced in single mode SOF, single mode POF, GIPOF-50 and the PMMA mPOF.

Figure 3 shows the linear regressions of the phase shifts induced in every type of optical fiber versus the peak pressure amplitudes produced by 1 and $10 \mathrm{MHz}$ emitters. The results are summarized in table 1 . For $1 \mathrm{MHz}$ the acoustic sensitivity for polymer optical fibers is thirteen times higher than for silica optical fiber. The mPOF extends this ratio to the $10 \mathrm{MHz}$ frequencies range, performing with 3 times more sensitivity than the other POFs. Moreover the attenuation of this fiber is low at $850 \mathrm{~nm}$ allowing the construction of longer sensors.

Table 1. Acoustic sensitivity for 1 and $10 \mathrm{MHz}$ emitters of single mode SOF, single mode POF (MORPOF03), GIPOF-50 and the PMMA microstructured POF.

\begin{tabular}{|l|l|l|l|l|}
\cline { 2 - 5 } \multicolumn{1}{c|}{} & SM-SOF $(\mathrm{mrad} / \mathrm{kPa})$ & SM-POF $(\mathrm{mrad} / \mathrm{kPa})$ & GIPOF-50 $(\mathrm{mrad} / \mathrm{kPa})$ & PMMA mPOF $(\mathrm{mrad} / \mathrm{kPa})$ \\
\hline $1 \mathrm{MHZ}$ & $0.72 \pm 0.01$ & $9.64 \pm 0.04$ & $9.57 \pm 0.11$ & $9.04 \pm 0.08$ \\
\hline $10 \mathrm{MHZ}$ & $0.64 \pm 0.03$ & $3.17 \pm 0.07$ & $3.12 \pm 0.12$ & $10.04 \pm 0.18$ \\
\hline
\end{tabular}

\section{CONCLUSION AND FUTURE WORK}

In this work we have demonstrated experimentally that the sensitivity in the detection of ultrasonic waves in the $\mathrm{MHz}$ range of interferometric $\mathrm{mPOF}$ sensor is greater one order of magnitude than for the traditional single-mode silica optical fibers. Moreover, compared to other POFs sensors, the mPOF has not only better sensitivity than GIPOF-50 all over the frequency spectrum, but can make enable interferometers with lower noise floor. One of the most promising avenues of the $\mathrm{mPOF}$ is the possibility to write fiber Bragg gratings on it. Future work will be centered in the use of mPOF with FBG and Fabry-Perot based on FBG for ultrasonic sensing.

\section{REFERENCES}

[1] K. Jansen, A. F. W. van der Steen, H. M. M. van Beusekom et al., "Intravascular photoacoustic imaging of human coronary atherosclerosis," Optics Letters, 36(5), 597-599 (2011).

[2] J.-M. Yang, C. Favazza, R. Chen et al., "Simultaneous functional photoacoustic and ultrasonic endoscopy of internal organs in vivo," Nature Medicine, 18(8), 1297 (2012).

[3] D. Gallego, and H. Lamela, "High-sensitivity ultrasound interferometric single-mode polymer optical fiber sensors for biomedical applications," Optics Letters, 34(12), 1807-1809 (2009).

[4] D. Gallego, and H. Lamela, "High sensitivity interferometric polymer optical fiber ultrasound sensors for optoacoustic imaging and biomedical application," Proceedings of SPIE. 7753, (2011). 